\title{
Integrated Bioinformatic Analysis Identifies TIPIN as a Prognostic Biomarker in Hepatocellular Carcinoma
}

\author{
Hui Chen $\mathbb{D}^{1},{ }^{1,2}$ Chunting Zhang, ${ }^{1,2}$ Qianmei Zhou, ${ }^{3}$ Yanan Guo, ${ }^{4}$ Zhigang Ren $\mathbb{D}^{1,2}$ \\ and Zujiang Yu iD ${ }^{1,2}$ \\ ${ }^{1}$ Precision Medicine Center, The First Affiliated Hospital of Zhengzhou University, Zhengzhou, China \\ ${ }^{2}$ Department of Infectious Diseases, The First Affiliated Hospital of Zhengzhou University, Zhengzhou, China \\ ${ }^{3}$ Department of Breast Surgery, The First Affiliated Hospital of Zhengzhou University, Zhengzhou, China \\ ${ }^{4}$ Department of Infectious Diseases, Zhengzhou Central Hospital, Zhengzhou, China
}

Correspondence should be addressed to Zujiang Yu; johnyuem@zzu.edu.cn

Received 9 June 2021; Accepted 23 December 2021; Published 17 January 2022

Academic Editor: Serena Del Turco

Copyright (c) 2022 Hui Chen et al. This is an open access article distributed under the Creative Commons Attribution License, which permits unrestricted use, distribution, and reproduction in any medium, provided the original work is properly cited.

Background. Gene expression and DNA methylation analyses have long been used to identify cancer markers. However, a combination analysis of the gene expression and DNA methylation has yet to be performed to identify potential biomarkers of hepatocellular carcinoma (HCC). Methods. By matching gene expression profiles and promoter methylation data in The Cancer Genome Atlas (TCGA), genes with discrepant expression as well as genes with differential promoter methylation were identified. High-expression genes with low promoter methylation were defined as epigenetically induced (EI), while low-expression genes with high promoter methylation were defined as epigenetically suppressed (ES). The human protein interaction network was further integrated to construct the EI/ES gene interaction network, and the key genes in the subnet were identified as potential HCC biomarkers. The expression differences and prognostic values were verified in TCGA and Gene Expression Omnibus (GEO) databases, as well as with tissue chip technology. Results. Four key genes were identified: TIPIN, RBM15B, DUSP28, and TRIM31, which demonstrated the differential gene expression and prognostic value in TCGA and GEO databases. Tissue microarray analysis (TMA) revealed that TIPIN levels were altered in HCC. The upregulated TIPIN expression was associated with worse overall survival. Univariate and multivariate analyses showed that the TIPIN expression was an independent predictor of HCC. Conclusion. TIPIN might be a potential novel prognostic biomarker for HCC.

\section{Introduction}

Liver cancer is the second leading cause of cancer-related deaths worldwide, with an increasing incidence in Asia and Africa [1]. Hepatocellular carcinoma (HCC) accounts for $70 \%-90 \%$ of liver cancers worldwide. Surgical resection provides the highest probability of long-term survival [2]; however, only $10 \%$ to $30 \%$ of patients are eligible for curative surgery because of late diagnosis [3], and the postoperative five-year survival rate is low [4]. The dismal clinical outcome of HCC is largely due to a particularly high rate of postsurgical recurrence and metastasis $[5,6]$. Thus, studies aimed at identifying novel HCC biomarkers to improve the early diagnosis rate and ultimately patient survival are needed.

Hepatocarcinogenesis is a complex multistep process involving the accumulation of genetic and epigenetic alterations [7]. Epigenetic regulation by promoter methylation plays a critical role in tumorigenesis [8]. Moreover, DNA epigenetic dysregulation signatures during tumorigenesis may be used as diagnostic or prognostic markers for cancer [9]. However, the DNA methylation patterns associated with gene expression and clinical prognosis in HCC remain to be elucidated.

In this study, promoter methylation and gene expression profiles from The Cancer Genome Atlas (TCGA) were used 


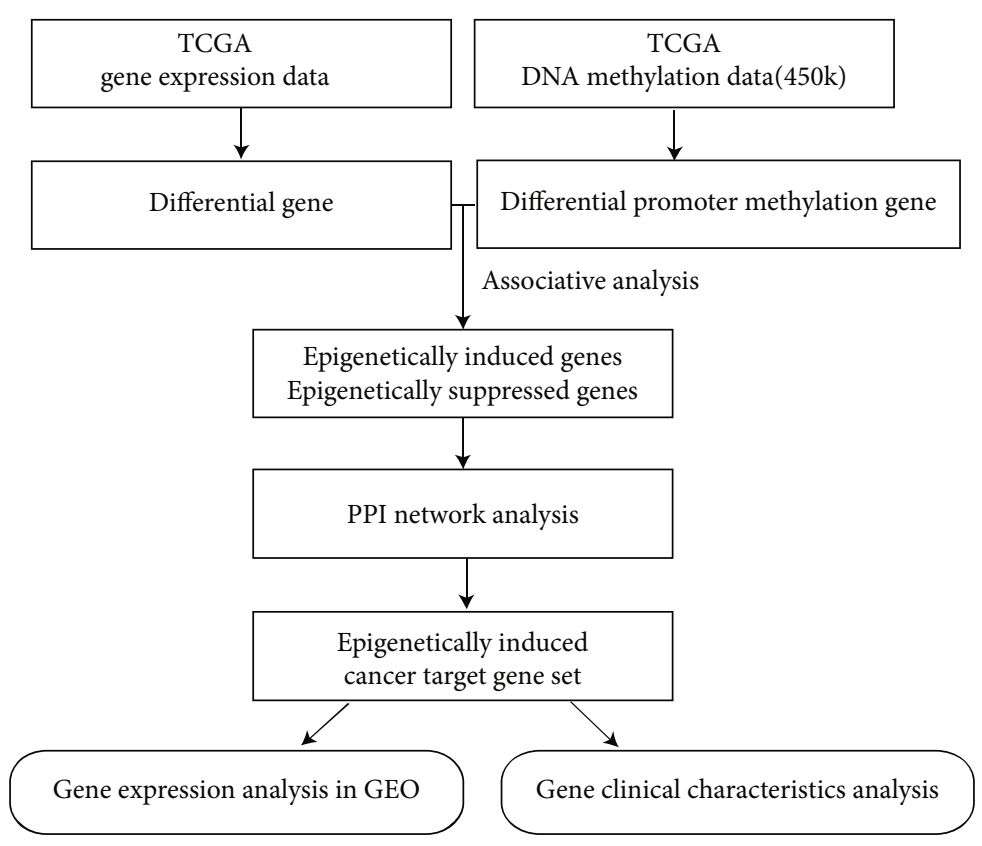

(a)

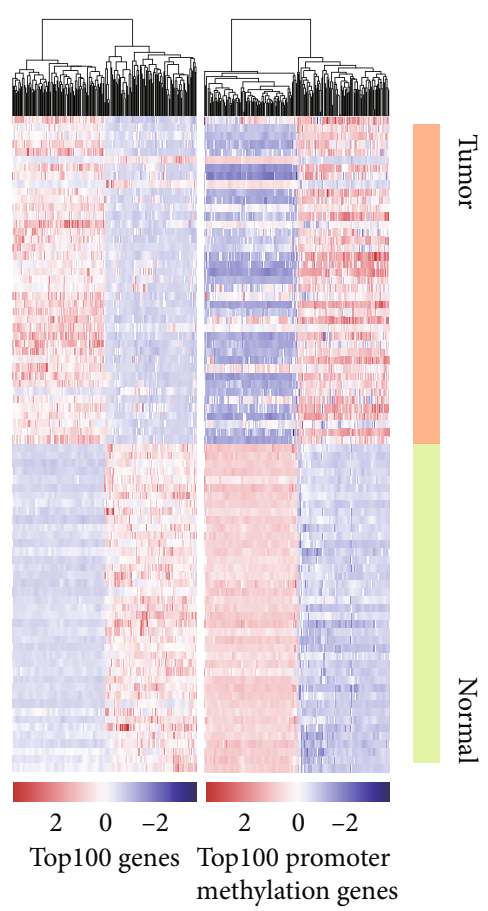

(b)

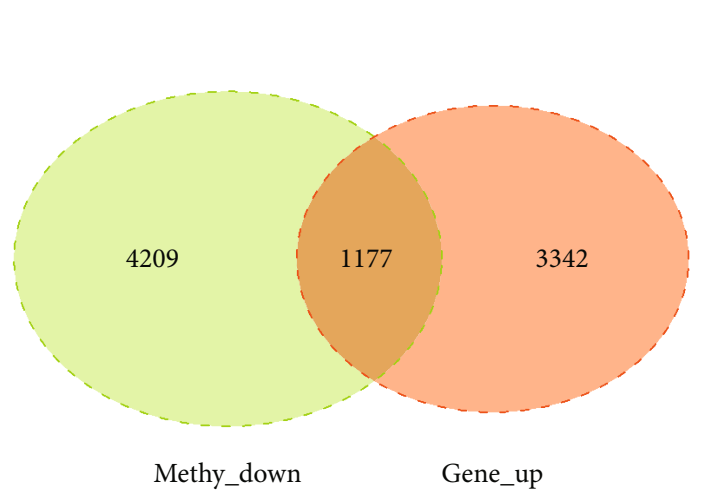

(c)

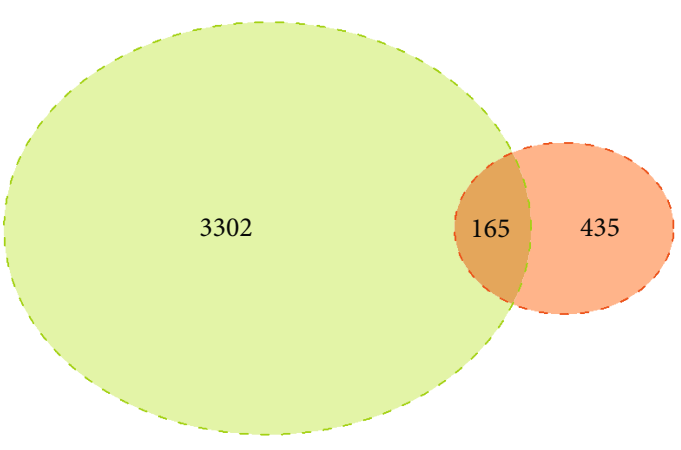

Methy_up

(d)

FIgUre 1: Continued. 

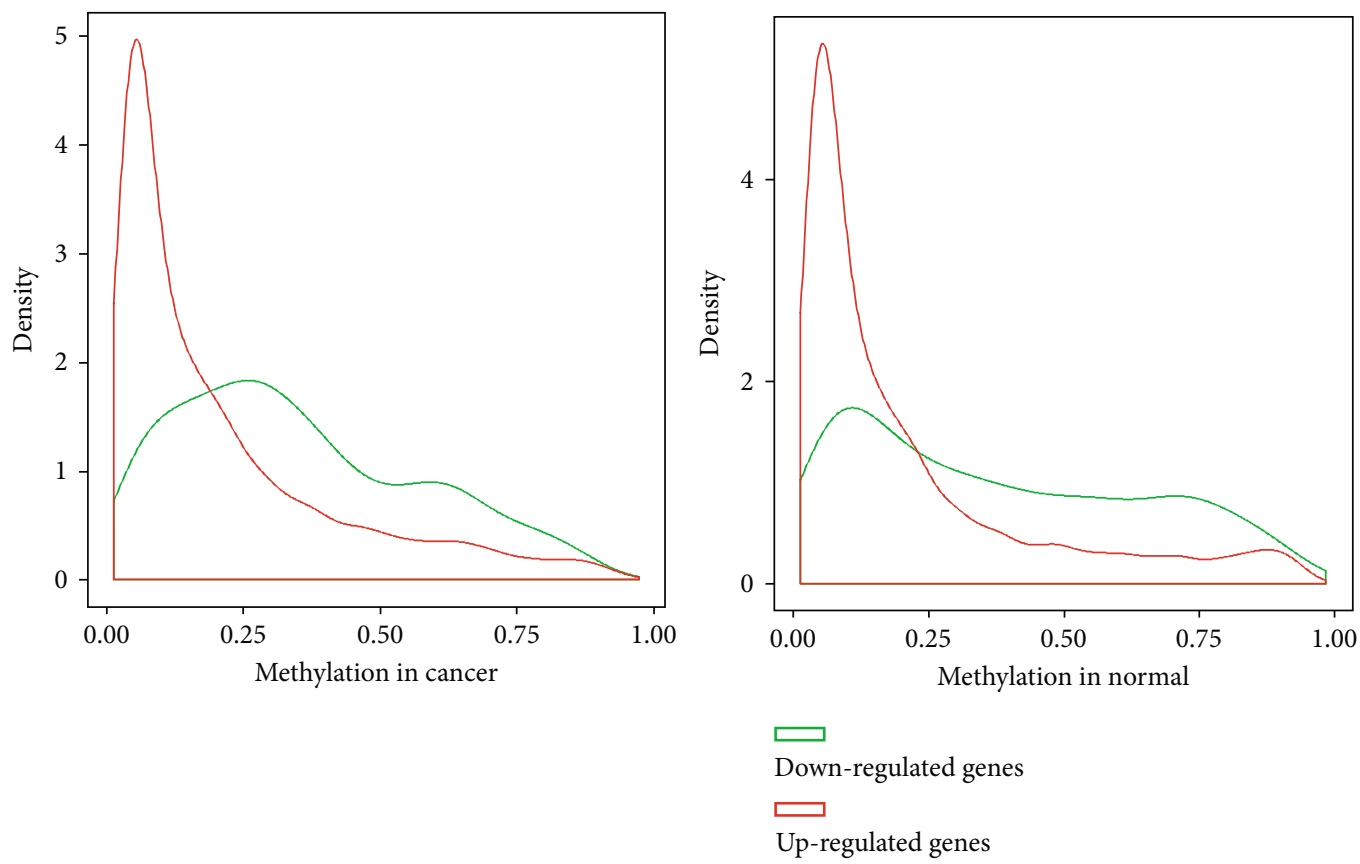

(e)

FIGURE 1: Integrated analysis of DNA methylation and gene expression profiles in TCGA. Notes: (a) The flowchart of the analysis process on selection of the epigenetically altered driver genes. (b) The cluster map of the mRNA expression of the top 100 genes and top 100 genes with promoter methylation in TCGA cohort. (c, d) Venn diagram of differentially expressed genes and genes with differential promoter methylation. (e) The negative relationship between gene expression and promoter methylation levels in cancer and normal tissues.

to screen candidate genes associated with HCC prognosis. We identified four genes: RNA binding motif protein $15 \mathrm{~B}$ (RBM15B), timeless- (TIM-) interacting protein (TIPIN), dual-specificity phosphatase 28 (DUSP28), and tripartite motif 31 (TRIM31). Subsequently, the expression difference was verified using Gene Expression Omnibus (GEO), and survival analysis was used to identify prognosis biomarkers of liver cancer. The relationship between these genes and HCC stages was validated using TCGA data, and subsequently, we focused on the gene TIPIN. Furthermore, the relationship between the gene expression of TIPIN and clinical features was demonstrated with a tissue microarray (TMA) of a cohort of patients with HCC $(n=160)$.

\section{Materials and Methods}

2.1. DNA Methylation and Gene Expression Profile Downloading and Processing. High-throughput data for RNA sequencing (RNA-seq) and DNA methylation and clinical characteristics of the liver cancer cohort were downloaded from TCGA (https://tcga-data.nci.nih.gov/tcga/). By matching methylation data and RNA-Seq data, 41 pairs of samples contained cancer, and paracancerous samples were obtained. Methylation data were measured on the $450 \mathrm{~K}$ array and quantified using beta values. The methylation probe was mapped to the promoter region of the gene, which was defined as $800 \mathrm{bp}$ upstream to $200 \mathrm{bp}$ downstream of TSS, and the empty probe was removed. When multiple probes corresponded to one gene, we used the average as the gene promoter methylation level. The RNA-seq data were measured by transcripts per million (TPM) transformed from fragments per kilobase million (FPKM) before the subsequent analysis.

2.2. Identification and Integration Analysis of Differential DNA Methylation and Gene Expression in HCC. The average methylation values at the $\mathrm{CpG}$ site and gene expression values were compared between the liver cancer and normal groups using Wilcoxon's test for nonpaired samples. The following criteria were used to calculate the differential expression: $\log 2$ (fold change) $>1$ and FDR-corrected $P$ value $<0.05$. To calculate the differential promoter methylation, we used $P$ value $<0.05$.

We defined genes with the high expression and low promoter methylation as epigenetically induced (EI) and genes with low expression and high promoter methylation as epigenetically suppressed (ES). We then analyzed the relationship between differentially expressed genes and genes with differential promoter methylation using a Venn diagram and computed Spearman correlation coefficients between promoter methylation level and expression for the candidate genes. Gene enrichment tests were performed on the candidate genes. ClusterProfiler (version 3.4.4) was used to detect significant enrichment for Gene Ontology (GO) and Kyoto Encyclopedia of Genes and Genomes (KEGG) pathways. All correlation analyses were performed using the Limma $R$ package 2.12.0. 


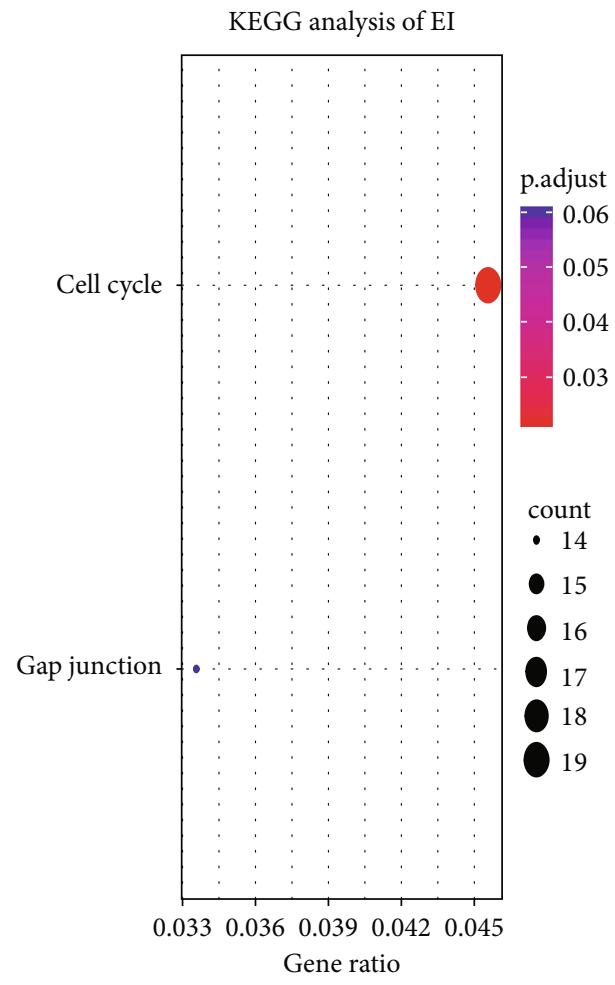

(a)

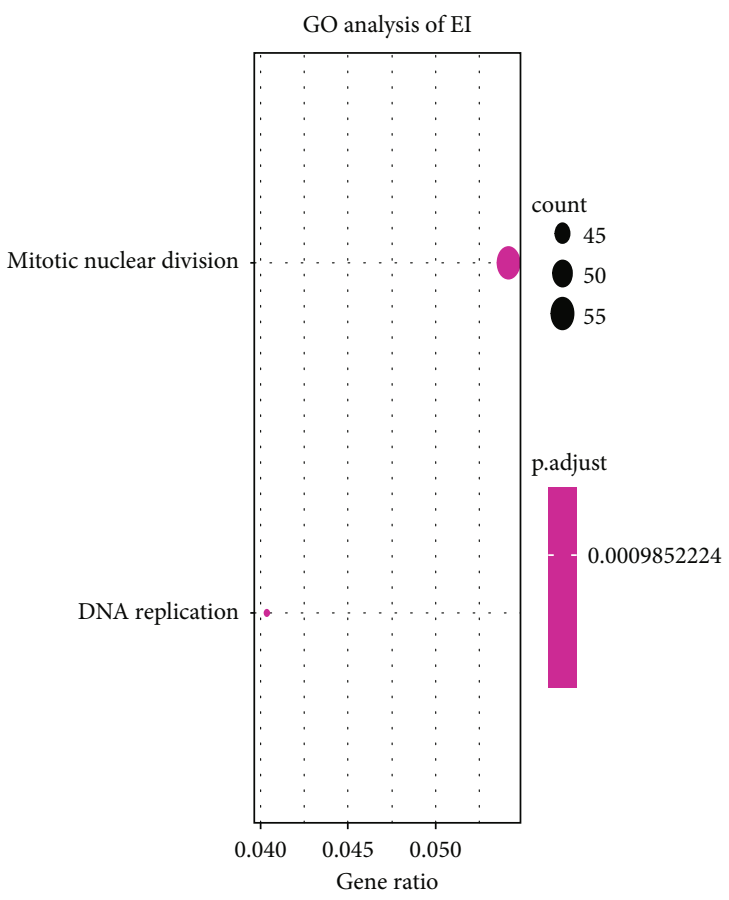

(c)

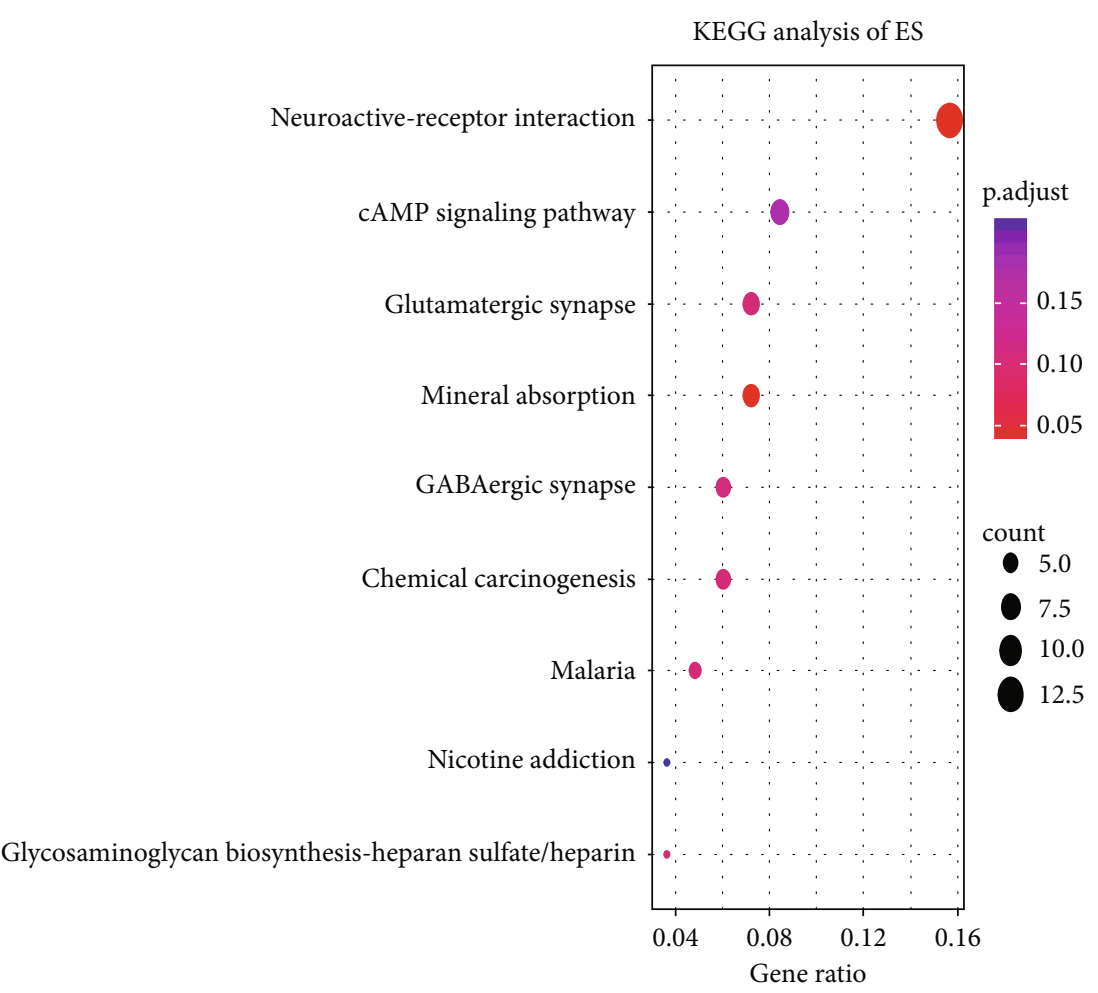

(b)

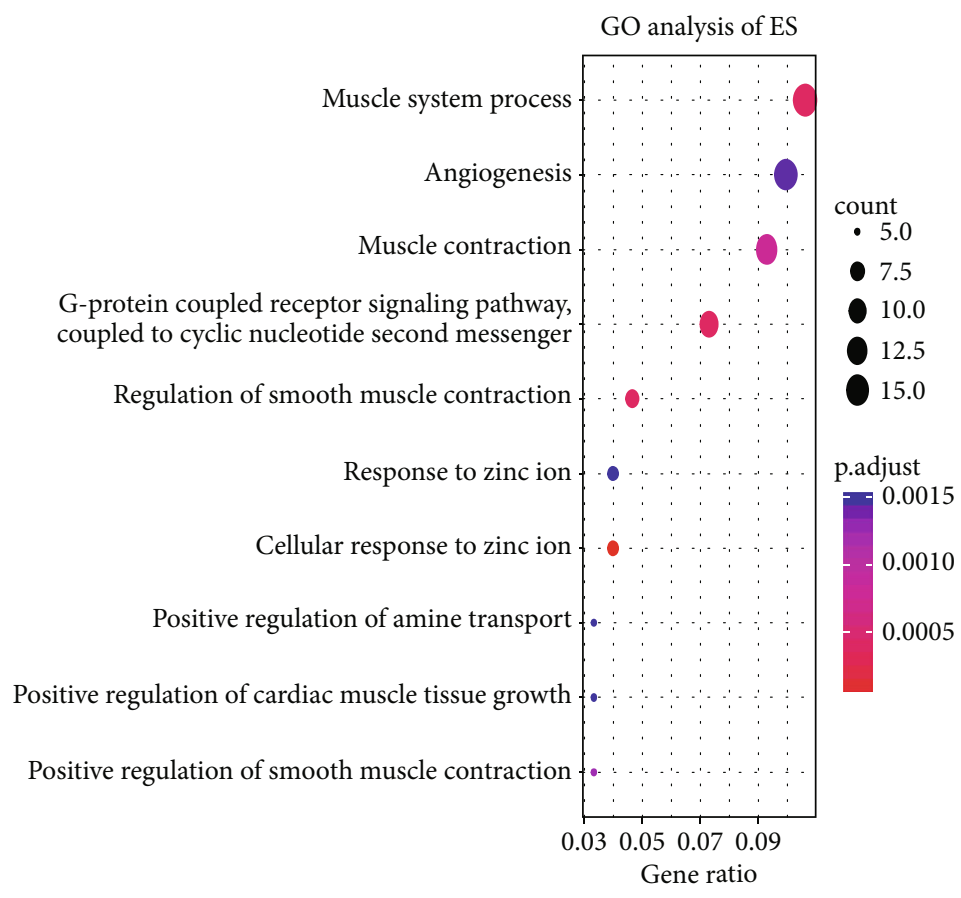

(d)

Figure 2: The consequence of KEGG and GO enrichment analysis of EI/ES genes in TCGA. Notes: (a) KEGG enrichment analysis of EI genes. (b) KEGG enrichment analysis of ES genes. (c) GO enrichment analysis of EI genes. (d) GO enrichment analysis of ES genes.

2.3. Interaction Control Network Construction and Analysis of Network Degree Distribution. Human protein interaction data were downloaded from the HIPPIE database (https://
www.ncbi.nlm.nih.gov/pmc/articles/PMC5210659) to construct a human protein interaction network. The obtained EI and ES genes were mapped into a human protein 


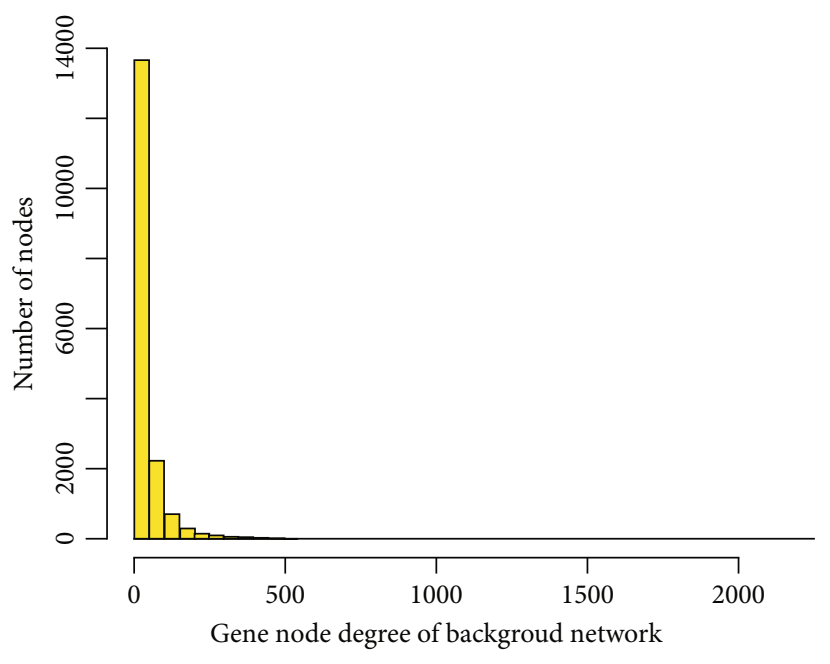

(a)<smiles></smiles>

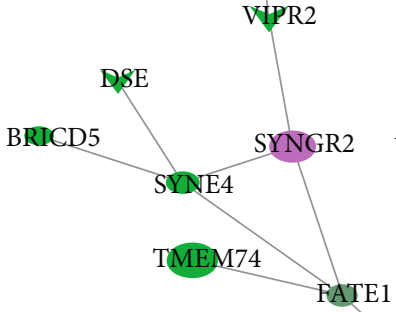

ATP6V1E2<smiles>[10BH2]</smiles>

ATP6V0D2

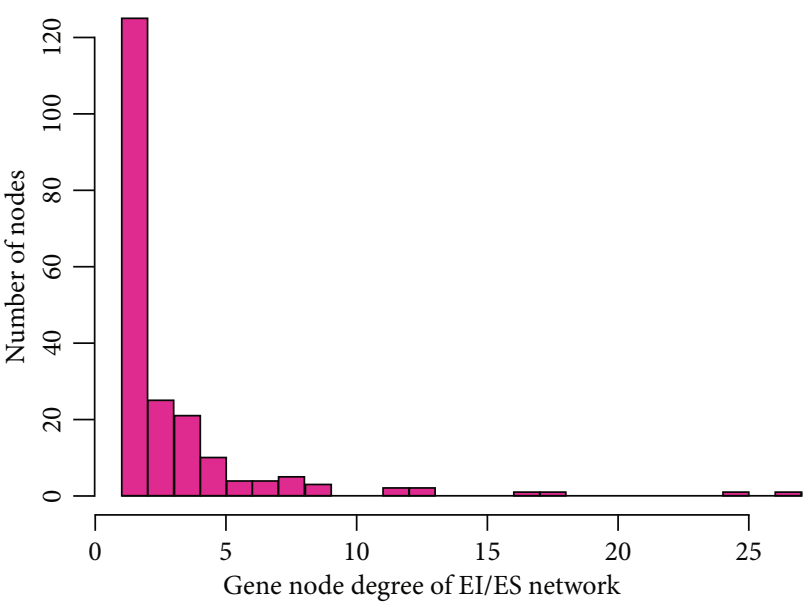

(b)

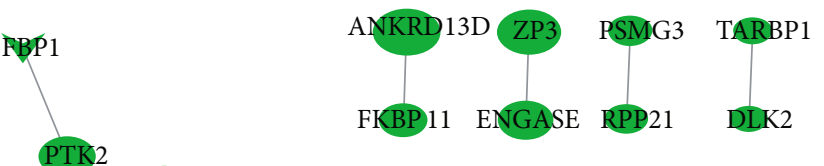

TKK2 ARR3

CUL9 PKD1 MEPE EGF

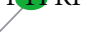

INE1A IL32

FUT1
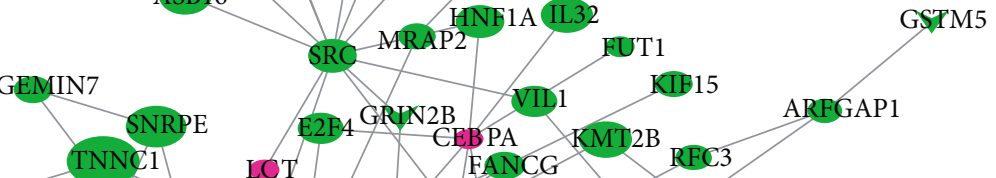

\section{3}

ARFGAP1

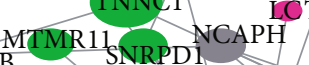

ADRA2B

ITPK1. CPA5 NHLRCI CDK1 TEXN1

TOPAT CURKB RFCI IRAK2

FAM 111A

TNFRSF4 DIEXI TOPIMT PRPF6 SMC4

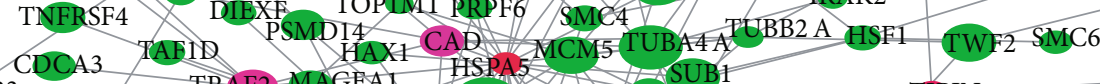

PYCR2

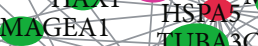

CCDC24 GDC23 GORASP2 TRAF2 MAGEA1 TUBA3C

PTTG1 TRIM3 H MKRN3 PSMD

PNMA1 RFPL4B GTPBP4 AAB1 HCM2RAD50 18 FOXRED2 COA

RRS1 PGP MVP IAPD 1 2 I IPO9 CSTAPIP5K1A

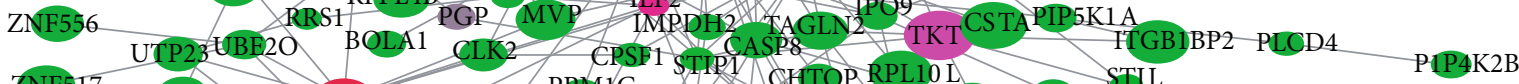
ZNF517 STAMBPL1 TRIM27 RBM28 PPMIG XIIPI CHTOP RPL10 L 15 RPAP1 STIL

TTLL10 PQDON5 FAM103A1 PDGFB PLEKHNI TCF20 VPS28

$$
\text { PGPEP1 }
$$

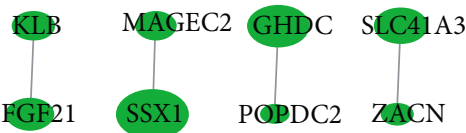
MRPL24 GMPS NUP43 DUSP28 EXOSC9

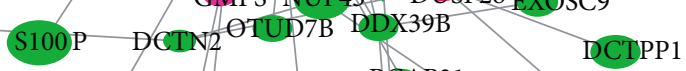

S100P DCTN2 BCAP31

BCAP31 TIPIN TIMLESS

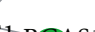

GINS1

2
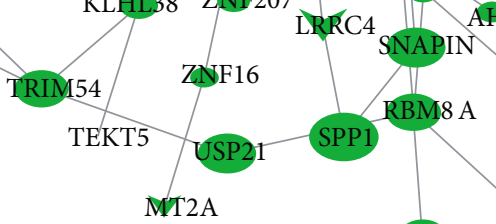
RBM8 A \$CAMP3

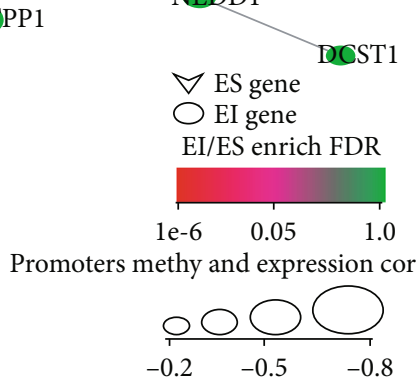

(c)

FIgURE 3: Degree distribution of network and EI/ES gene interaction network. Notes: (a) Degree distribution of background network. (b) Degree distribution of EI/ES gene interaction network. (c) EI/ES gene interaction network. 
TABLE 1: The selected epigenetically altered driver.

\begin{tabular}{|c|c|c|c|c|c|c|c|}
\hline Gene symbol & $\begin{array}{l}\text { Number of EI } \\
\text { neighbor gene }\end{array}$ & $\begin{array}{c}\text { Number of } \\
\text { neighbor gene }\end{array}$ & $\begin{array}{l}\text { Number of } \\
\text { EI gene }\end{array}$ & $\begin{array}{c}\text { Number of } \\
\text { network gene }\end{array}$ & $\begin{array}{l}\text { EI neighbor } \\
\text { gene per }\end{array}$ & $\begin{array}{l}\text { Fisher's exact } \\
\text { test } P \text { value }\end{array}$ & FDR \\
\hline TIPIN & 4 & 12 & 205 & 17381 & 0.250 & $3.06 E-05$ & 0.006 \\
\hline RBM15B & 5 & 31 & 205 & 17381 & 0.139 & $6.09 E-05$ & 0.012 \\
\hline DUSP28 & 5 & 26 & 205 & 17381 & 0.161 & $2.88 E-05$ & 0.006 \\
\hline TRIM31 & 4 & 20 & 205 & 17381 & 0.167 & $1.66 E-04$ & 0.033 \\
\hline
\end{tabular}

TIPIN: TIM-interacting protein; RBM15B: RNA binding motif protein 15B; DUSP28: dual specificity phosphatase 28; TRIM31: tripartite motif 31; EI: epigenetically induced genes; FDR: false discovery rate.

interaction network to construct an EI/ES gene interaction subnetwork.

We set the human protein interaction network as the statistical background and counted the number of genes and EI/ES genes interacting with each EI and ES genes. We then constructed a statistical model using Fisher enrichment testing for each EI/ES gene. The following criteria were used to select the key epigenetically altered driver genes: (1) FDR $<0.05$ and (2) the proportion of EI/ES genes in the neighbor node of the gene was more than $10 \%$.

2.4. GEO Dataset Processing and Analysis. Twenty sets of microarrays were obtained from the GEO database (http; //http://www.ncbi.nlm.nih.gov/geo/) and were used to demonstrate target gene expression. The 20 cohorts were compounded of GSE6764, GSE14520, GSE36376, GSE39791, GSE45436, GSE54236, GSE54238, GSE57957, GSE60502, GSE62232, GSE64041, GSE76297, GSE76427, GSE25097, GSE77314, GSE84005, GSE84598, GSE102083, GSE10143, and GSE14811. The characteristics including cohort ID, RNA-seq platform, number of samples (cancer and noncancer samples), publication year, and country are summarized in Table S1.

2.5. Patients and Specimens. TMAs containing 80 pairs of HCC specimens and corresponding nontumor tissues obtained from the First Affiliated Hospital of Zhengzhou University in 2015 to 2019 were constructed using a $1.5 \mathrm{~mm}$ diameter core. All specimens were collected from patients who underwent surgical resection at the Hospital, and all pathological data were retrieved from the pathology department. Diagnosis was confirmed by two pathologists, according to the American Joint Committee on Cancer TNM staging classification for carcinoma of the liver $\left(7^{\text {th }}\right.$ ed., 2011). We have obtained the approval of the Institutional Review Board of the First Affiliated Hospital of Zhengzhou University. Written informed consent was obtained from each patient, and this study complied with the Declaration of Helsinki.

2.6. Immunohistochemical Staining. TMA sections, with $5 \mu \mathrm{m}$ thickness, were dewaxed, treated with hydrogen peroxide to quench endogenous peroxidase activity, and then incubated with rabbit anti-human antibody $(1: 100$; Proteintech, Wuhan, China) at $4^{\circ} \mathrm{C}$ overnight. Biotinylated goat anti-rabbit secondary antibodies (1:200; Proteintech,
Wuhan, China) were then added. Finally, the samples were treated with $\mathrm{DAB}$ solution for $3 \mathrm{~min}$ to visualize the staining. Cells containing brown granules were independently counted by two pathologists who were blinded to the clinical parameters. According to the staining intensity, the samples were scored as 1 , weak; 2 , moderate; 3 , strong; and 4 , intense. Scores of 1-2 and 3-4 were defined as low expression and high expression, respectively.

2.7. Statistical Analysis. Statistical analysis was performed using SPSS software (version 25.0) or $R$ (version 3.6.1). The Wilcoxon matched-pair signed-rank test was used to compare protein expression differences between carcinoma and paracancerous tissues of HCC patients. The chisquared test was used to determine the association between gene expression levels and clinicopathological characteristics. The Kaplan-Meier method was used for survival analysis. Univariate and multivariate Cox proportional hazard regression models were used to analyze prognostic factors. Pearson's correlation coefficient was used to assess the linear association between the two variables.

\section{Results}

3.1. Integrated Analysis of DNA Methylation and Gene Expression Profiles. As shown in the analysis flow chart in Figure 1(a), we obtained RNA-seq and methylation data for 41 pairs of cancer and paracancerous samples from TCGA datasets. There are 17,937 genes that showed promoter methylation. From this gene set, we obtained 5,119 genes that showed the discrepant gene expression in cancer and adjacent cancer samples, according to the principle of differential screening. Of these, 4,519 genes were upregulated, and 600 genes were downregulated. Moreover, promoter methylation of 8,853 genes was differentially expressed in cancer and paracancer samples; of these genes, 3,467 promoter methylation were upregulated, and 5,386 were downregulated. The top 100 most distinguishing genes are shown in Figure 1(b). It is well known that DNA promoter methylation and gene expression have a negative correlation, as high methylation level inhibits the expression of the downstream gene, and low methylation level promotes the gene expression [10]. Therefore, we defined two concepts: low promoter methylation and high expression gene were epigenetically induced gene (EI); high promoter methylation and low 


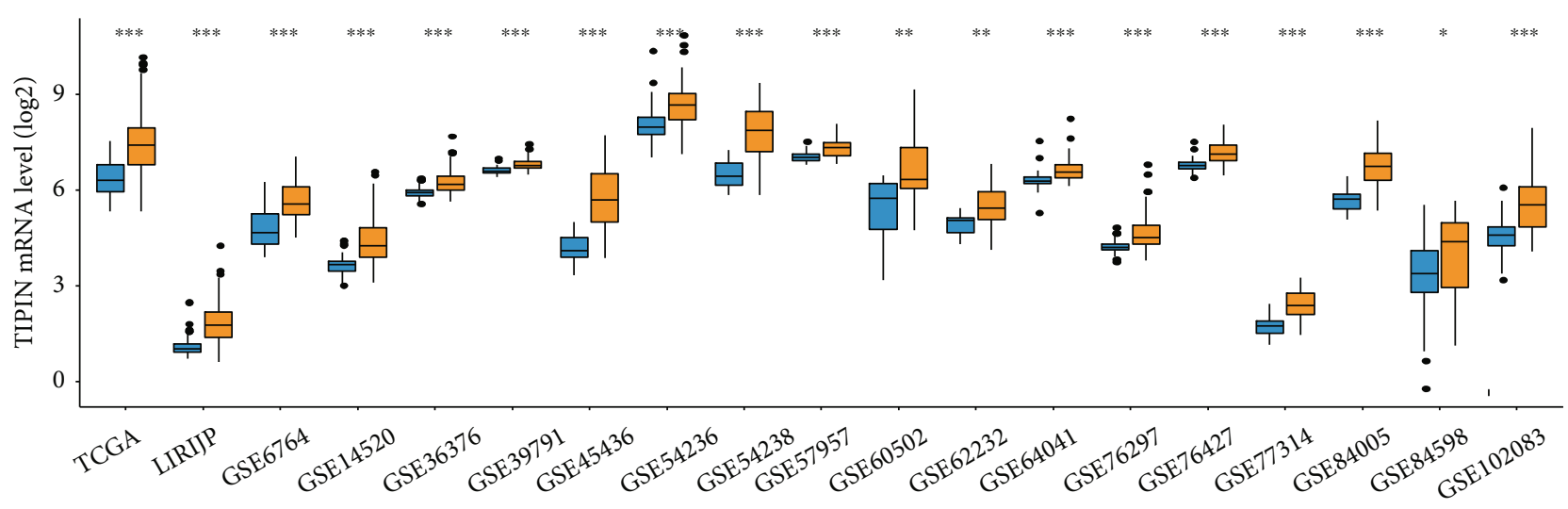

Normal

它 Tumor

(a)
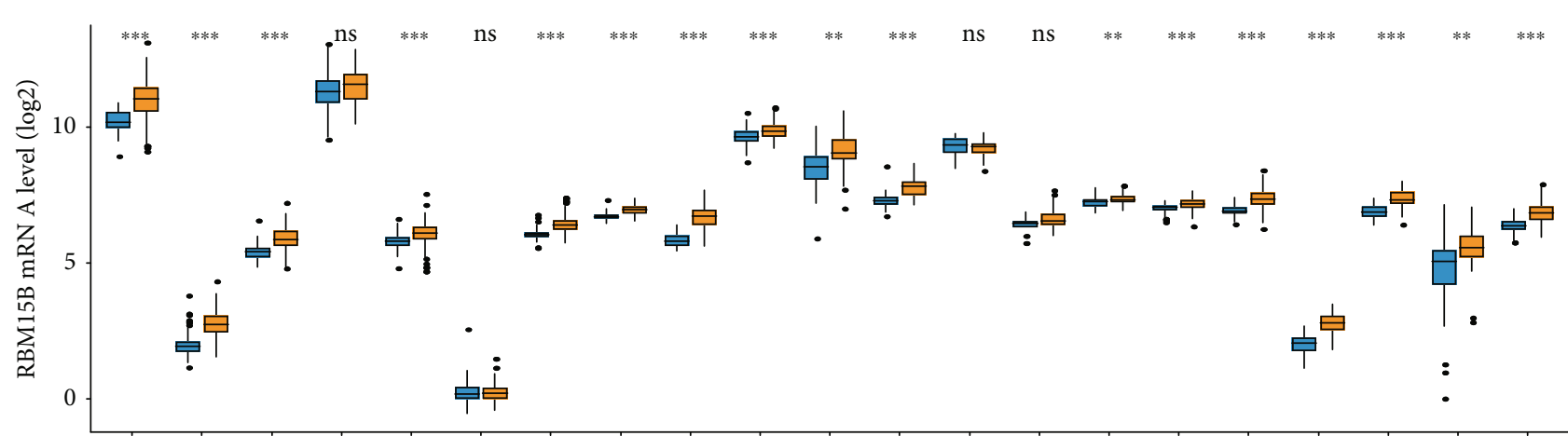

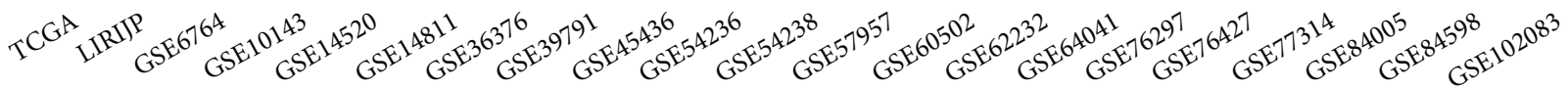

官 Normal

白 Tumor

(b)

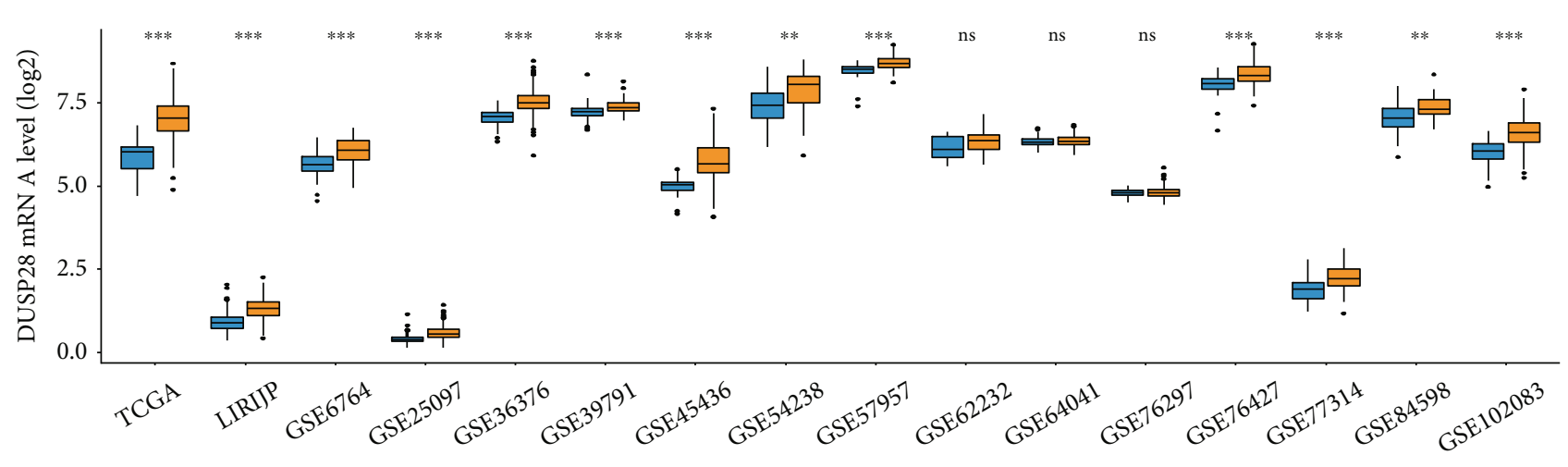

㝏 Normal

白 Tumor

(c)

FIgURE 4: Continued. 


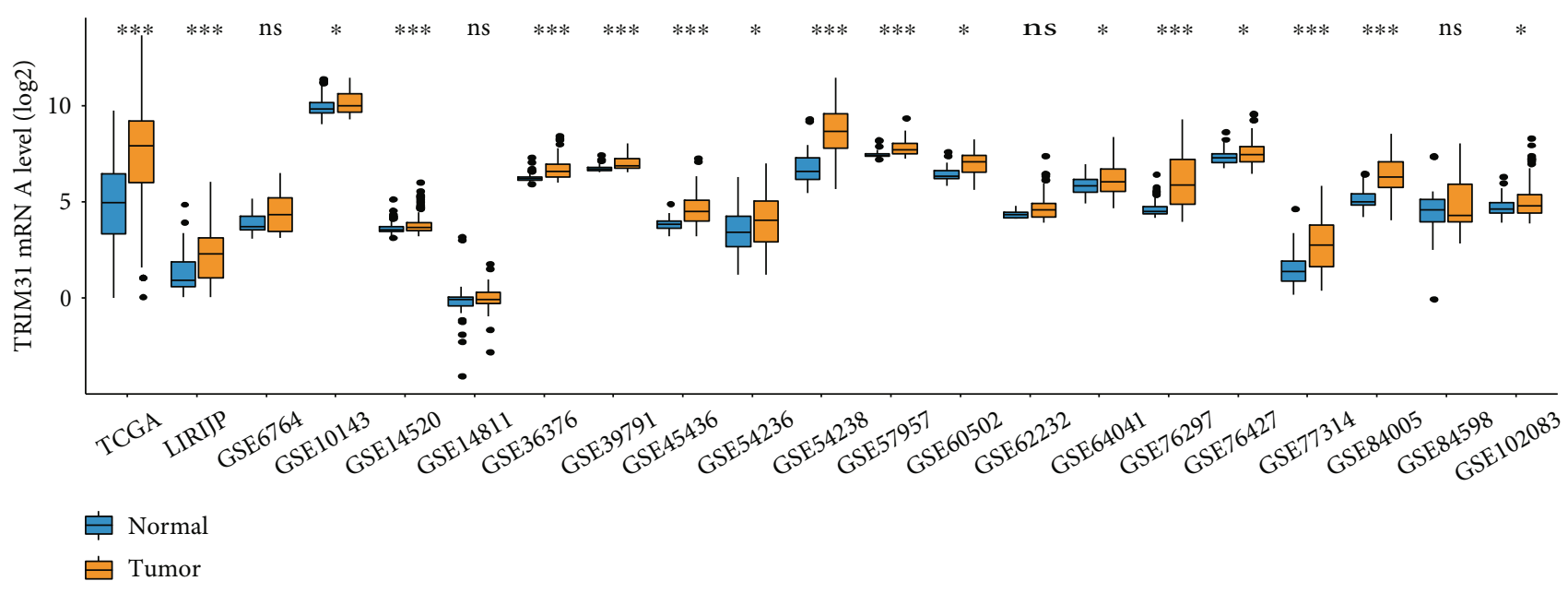

(d)

FIGURE 4: Expression of four expression-specific mRNAs in databases. Notes: The mRNA expression of (a) TIPIN, (b) RBM15B, (c) DUSP28, and (d) TRIM31 in databases. ${ }^{*} P<0.05,{ }^{* *} P<0.01,{ }^{* * *} P<0.001,{ }^{\text {ns }} P>0.05$.

expression gene were epigenetically suppressed gene (ES). The Venn diagram in Figures $1(\mathrm{c})$ and 1(d) shows 1,177 EI and 165 ES genes by combining genes with differential promoter methylation and differentially expressed genes. Figure 1(e) shows that the higher the promoter methylation level in cancer and normal tissues, the lower the expression of differentially expressed genes, indicating that promoter DNA methylation was negatively correlated with the gene expression.

3.2. Selection of the Epigenetically Altered Driver Genes. KEGG and GO enrichment analyses were performed to elucidate the biological functions of 1,177 EI and 165 ES genes (Figure 2). We observed that EI genes mainly gather in the cell cycle, gap junction, DNA replication, and mitotic nuclei, which have been reported to be associated with the occurrence of cancer (Figures 2(a) and 2(c)). Conversely, ES genes mainly gather in mineral absorption, glutamatergic synapse, GABAergic synapse, and cAMP signaling pathway, which are necessary for normal cell function and integrity (Figures 2(b) and 2(d)). By calculating the correlation of EI or ES genes between gene expression and promoter methylation, we obtained $419 \mathrm{EI}$ and $68 \mathrm{ES}$ genes with a negative correlation. Moreover, we loaded human protein interaction data from the HIPPIE database to construct protein interactive networks, including 17,381 nodes and an average of 19.6 neighbor nodes. Furthermore, EI and ES genes were mapped to the human protein internetworks to structure EI/ES gene interaction networks, where 436 genes were mapped to the network and included 315 nodes, with an average of 1.54 neighbor nodes. Figure 3(c) shows that the number of ES genes are few than EI genes, and the enrichment degree of the EI/ES genes is mostly low (the green circles are more). Finally, we chose TIPIN, RBM15B, DUSP28, and TRIM31 (Table 1) as epigenetically altered driver genes, in which the proportion of EI/ES genes in the neighbor node of the gene was more than $10 \%$, and the FDR of $P$ value in the enrichment significance of EI/ES genes was less than 0.05 . As shown in Figure S1A, a high correlation was found between promoter methylation level and expression of the expression-specific genes. Additionally, DUSP28 and TRIM31 have been reported to be associated with liver cancer.

3.3. The Prognostic Value of the Epigenetically Altered Driver Genes Determined Using TCGA and GEO. To explore the prognostic value of the four genes, data were retrieved from TCGA and GEO. The mRNA expression data were used to determine differential gene expression (Figure 4). The results show that the mRNA expression of the four genes is significantly upregulated in cancer samples compared to normal samples. Additionally, we analyzed the correlation between gene expression and the survival rate (Figure 5). The results suggest that all four expression-specific genes might be prognostic biomarkers of liver cancer.

To observe the changes in expression of the four genes during the development of cancer, we used TCGA data to analyze their expression at different stages (Figure 6(a)). RBM $15 B$ and TIPIN showed significant differences in the stages of cancer development. Moreover, during the progression of liver disease, the TIPIN mRNA expression was subsequently increased, as shown in Figure 6(b). This suggests that TIPIN may promote the progression of liver disease. Meanwhile, patients with an advanced TNM stage and high TIPIN expression had a poor prognosis (Figure 6(c)). Overall, our results showed that the four specific genes, TIPIN, RBM15B, DUSP28, and TRIM31, were likely prognostic biomarkers of liver cancer, and TIPIN might conspicuously accelerate the process of hepatocarcinogenesis. In addition, the results of ROC analysis revealed a significant diagnostic value of TIPIN in HCC (Figure S2).

3.4. Upregulated TIPIN Is Associated with Clinicopathological Characteristics. To further verify the prognostic value of 


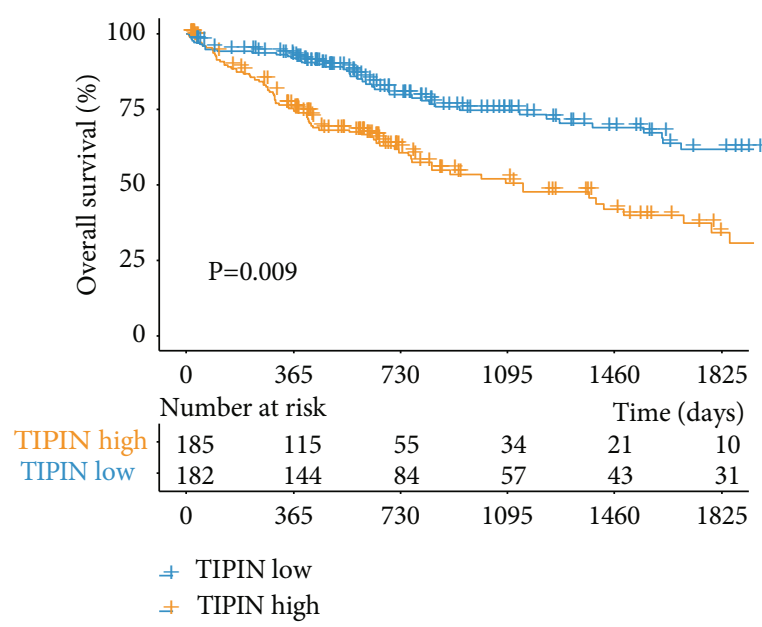

(a)

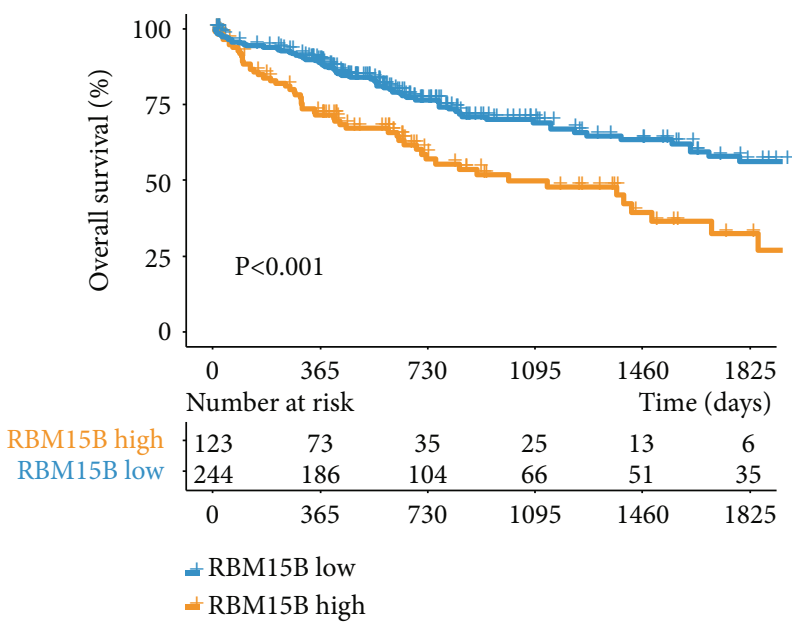

(c)

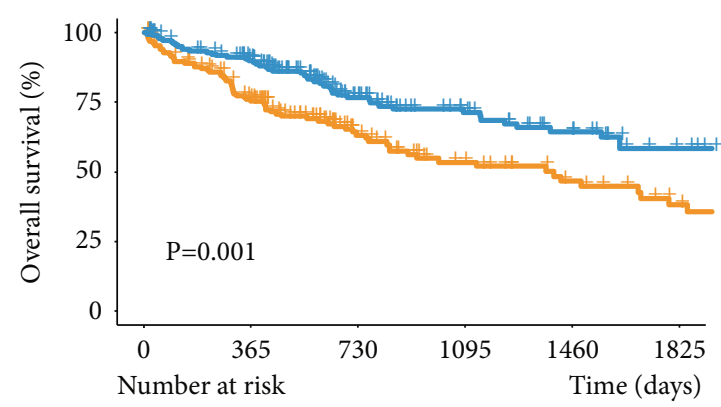

\begin{tabular}{l|lllll} 
DUSP28 high & 176 & 112 & 57 & 36 & 25
\end{tabular}

DUSP28 low \begin{tabular}{cccccc}
191 & 147 & 82 & 55 & 39 & 26 \\
\hline 0 & 365 & 730 & 1095 & 1460 & 1825
\end{tabular}

\pm DUSP28 low

\pm DUSP28 high

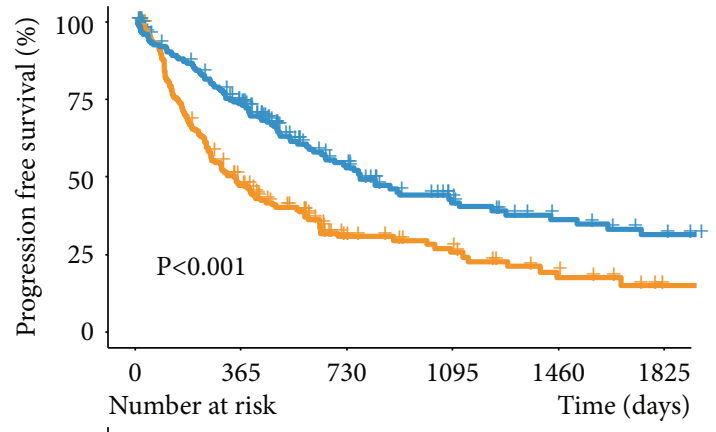

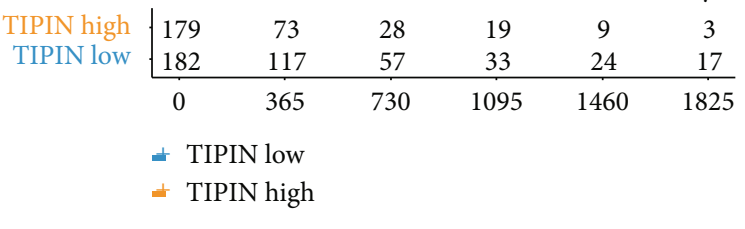

(b)

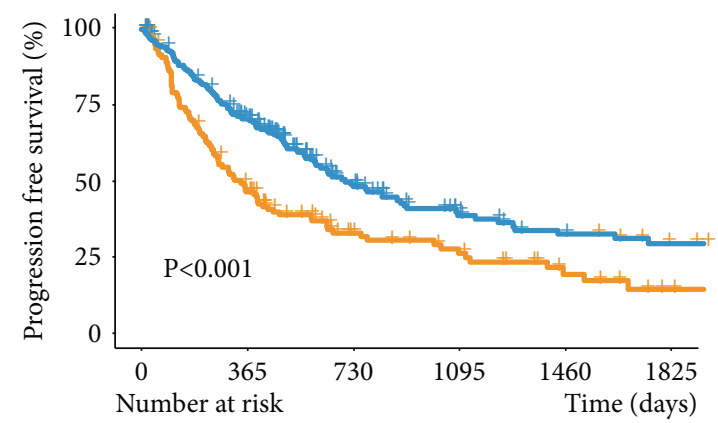

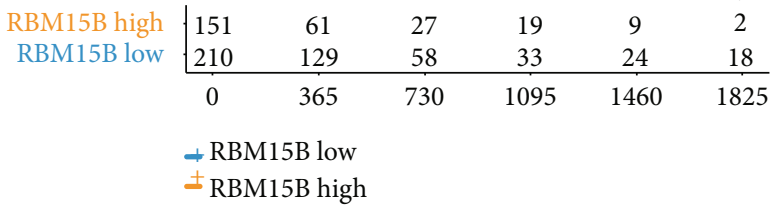

(d)

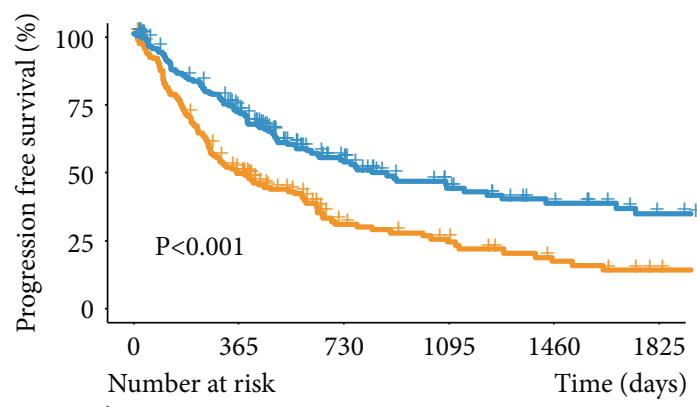

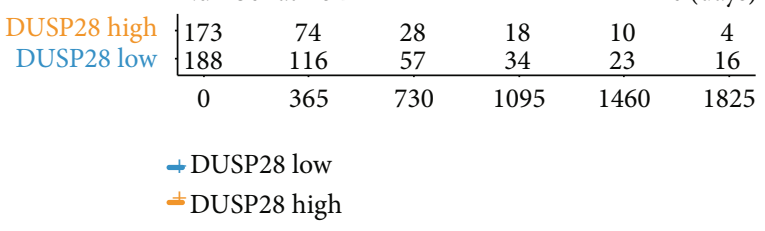

(f)

FIGURe 5: Continued. 


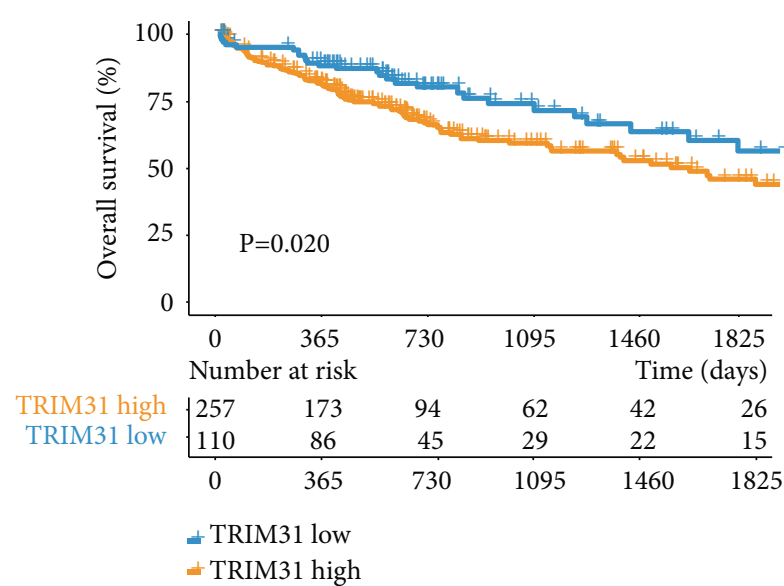

(g)

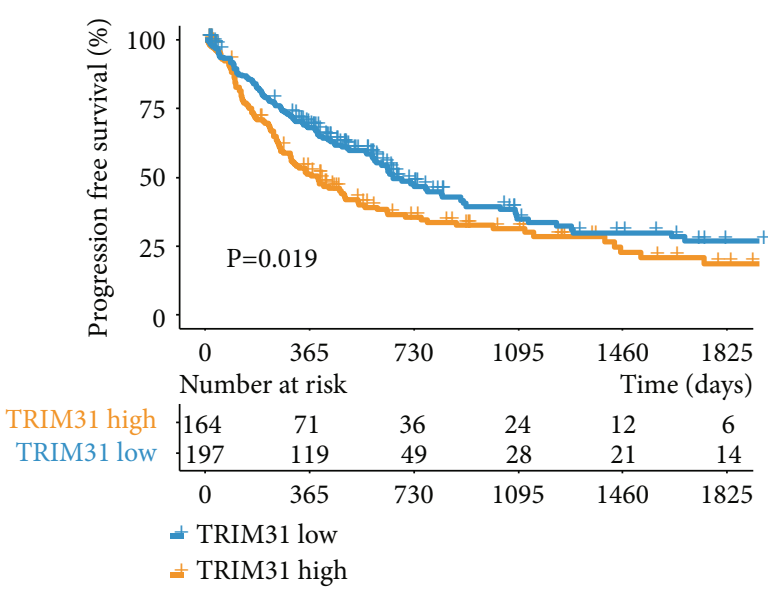

(h)

FIgURE 5: Survival analysis based on mRNA expression of four expression-specific genes. Notes: (a) OS analysis with the TIPIN expression. (b) PFS analysis with the TIPIN expression. (c) OS analysis with the RBM15B expression. (d) PFS analysis with the RBM15B expression. (e) OS analysis with the DUSP28 expression. (f) PFS analysis with the DUSP28 expression. (g) OS analysis with the TRIM31 expression. (h) PFS analysis with the TRIM31 expression.

TIPIN for HCC, 80 pairs of cancer and paracancerous samples and associated clinical information were collected. Tissue samples were used for immunohistochemical staining, and clinical data were used for correlation analysis and survival analysis. According to the staining intensity, TIPIN staining was scored from 1 to 4 (Figure 7(a)). A score of 1 to 2 was defined as the low TIPIN expression, whereas a score of 3 to 4 was defined as the high TIPIN expression. The results showed that the TIPIN level was altered between cancer and paracancerous tissues (Figures 7(b) and 7(c)). High TIPIN levels were associated with poor prognosis, based on the overall survival (OS) analysis $(P=0.011$, Figure $7(\mathrm{~d}))$. We then analyzed the relationship between TIPIN levels and clinical characteristics, and the results indicated that TIPIN levels were correlated with TNM stages (Table 2). Univariate and multivariate survival analyses showed that TIPIN levels and TNM stage were independent prognostic factors for HCC (Table 3 and Table S2). In addition, we conducted gene set enrichment analysis (GSEA) based on the Molecular Signatures Database (MSigDB) to preliminarily explore the molecular mechanism of TIPIN in HCC progression (Figure S3).

\section{Discussion}

DNA methylation drives epigenetic regulation by methyltransferase-mediated catalysis from CG nucleotide cytosine to 5-methylcytosine [11]. Dysfunction of DNA methylation can lead to activation of oncogenes and inactivation of tumor suppressor genes; abnormal DNA methylation is often acted as a characteristic of malignant tumors $[12,13]$. In comparison to genetic mutations, DNA methylation is a more potential therapeutic target in malignancies, because of the reversibility of epigenetic modifications [14]. Currently, many drugs like 5-azacytidine (5-AZA) and dec- itabine are in clinical trials in HCC, which may alter DNA methylation patterns or levels [15]. Previous studies have shown that abnormal DNA methylation is closely related to the occurrence, diagnosis, prognosis, and treatment of liver cancer $[16,17]$. For example, DNA methylation interferes with the expression of the deleted in the liver cancer 1 (DLC-1) gene, leading to the initiation of HCC as the gene encodes a tumor suppressor [18]. Other genes encoding tumor suppressors could also be silenced by DNA methylation, such as Ras association domain family 1A (RASSF1A) [19], human runt-related transcription factor 3 (RUNX3) [15], multiple tumor suppressor 1 ( $p 16)$ [20], and suppressor of cytokine signaling 1 (SOCS-1) $[21,22]$. The expression of the gene encoding Bcl-2-like protein $10(B C L B)$ could be reduced by DNA methylation, and the decreased $B C L B$ expression might be a therapeutic target for HCC because the gene is involved in HCC development [23]. It is reported that after sorafenib treatment-the only treatment option for unresectable and advanced HCC, oncogenes were prone to hypermethylation, and the tumor suppressor genes were apt to be hypomethylated in human-derived hepatoma cell line (HA22T/VGH), such as the hypermethylation of Janus kinase (JAK1) gene and the hypomethylation of SMAD family member 2 (SMAD2) gene [24]. Abovementioned all indicate that abnormal DNA methylation plays a coordinating role in promoting the initiation and development of HCC. Moreover, DNA methylation biomarkers may become targets for the diagnosis and treatment of HCC.

There are few studies on the combination of DNA methylation analysis with gene expression to search for tumor molecular markers. In our study, integrated analysis of gene expression and DNA methylation identified four genes: TIPIN, RBM15B, DUSP28, and TRIM31. As a member of the atypical DUSP family, DUSP28 has been reported to be significantly upregulated in HCC tissues and cell lines and 

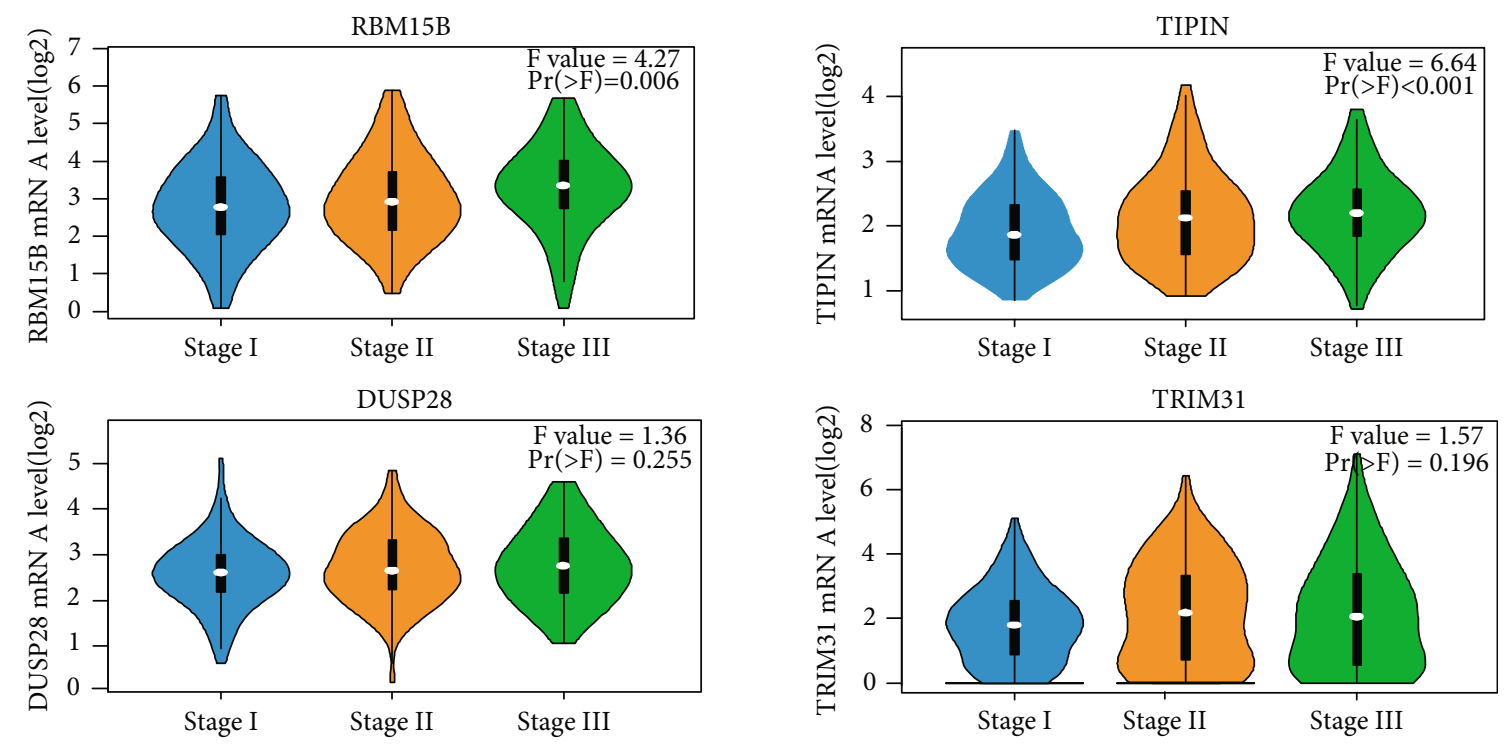

(a)
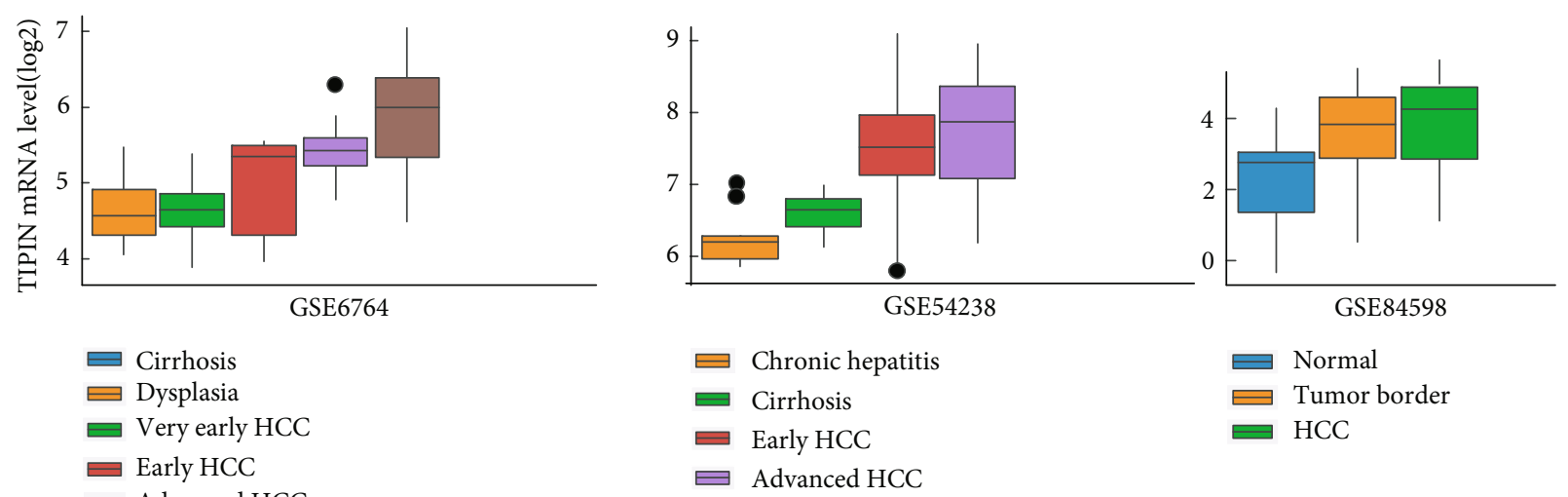

$\varpi$ Cirrhosis
$\square$ Dysplasia
$\square$ Very early HCC
$\square$ Early HCC
$\equiv$ Advanced HCC
$\square$ Very advanced HCC

(b)
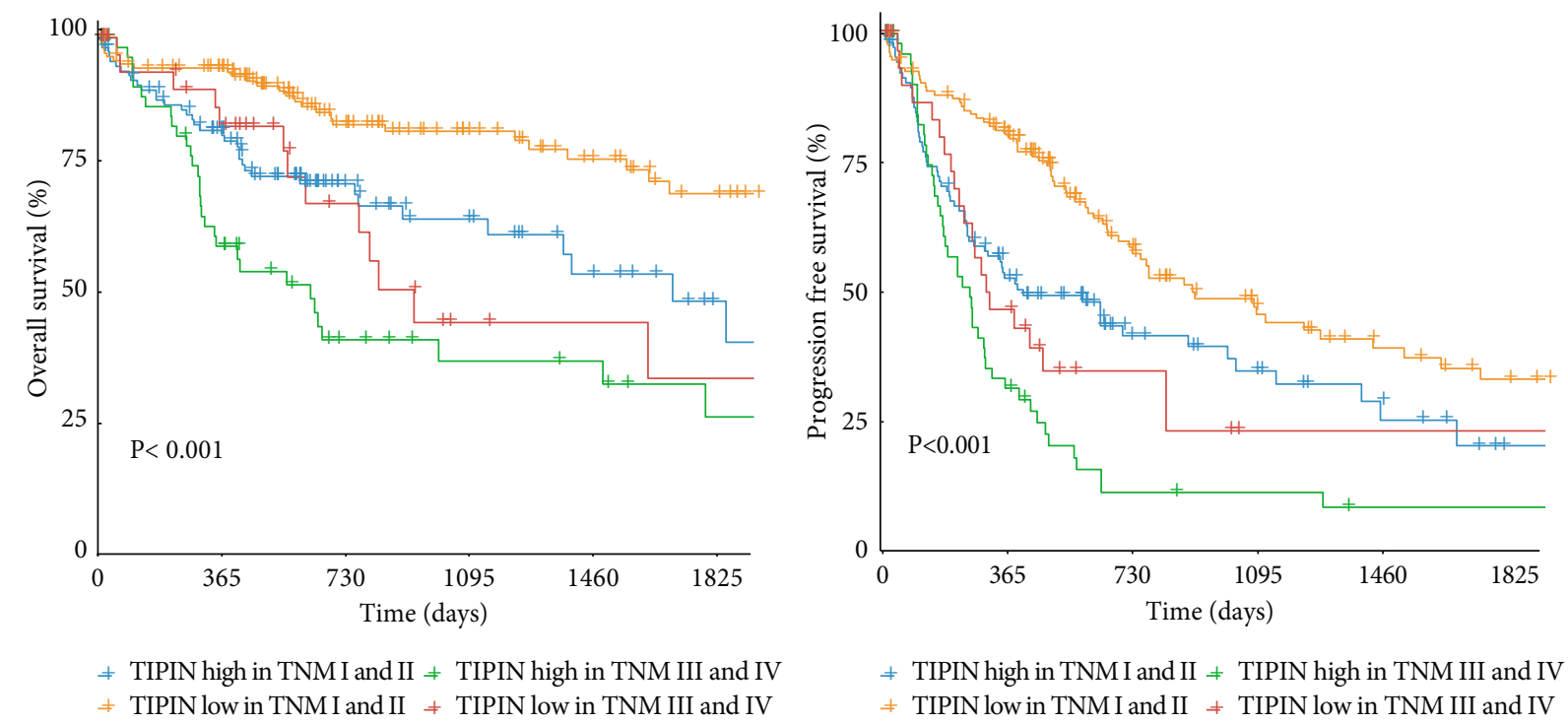

(c)

FIgURE 6: The expression of four expression-specific mRNAs during the development of HCC. Notes: (a) Expression of four specific mRNAs during different stages of liver cancer in TCGA. (b) The TIPIN expression during the development of liver disease in GEO databases. (c) Overall survival analysis of patients with different TNM stages, based on the TIPIN expression in TCGA. 

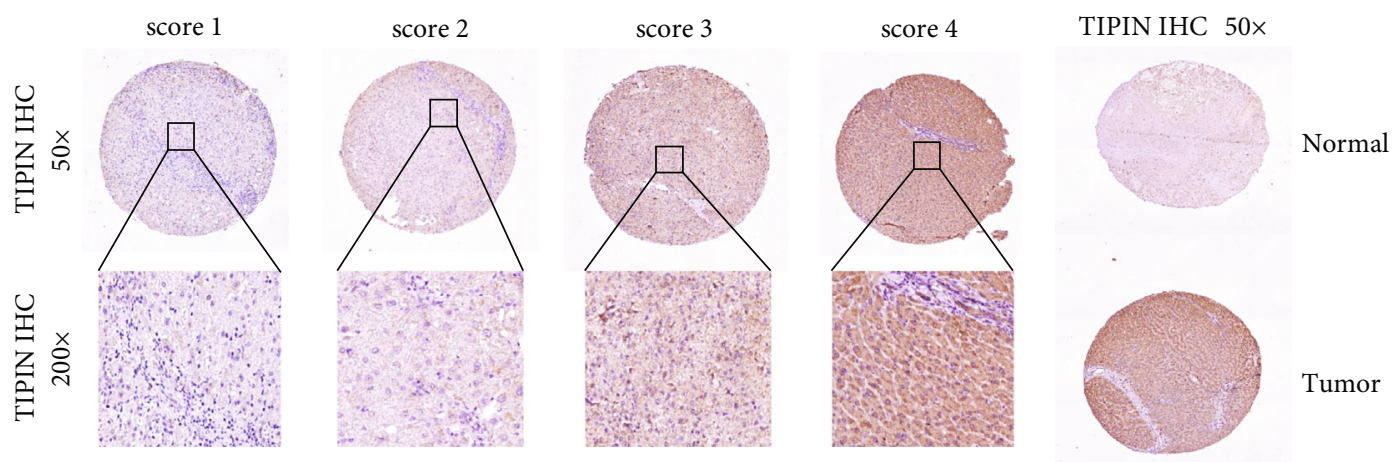

(a)

(b)

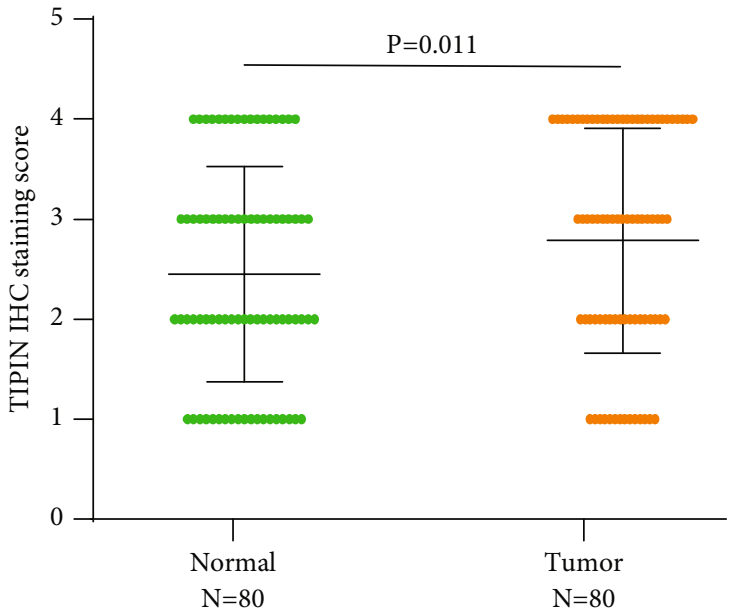

(c)

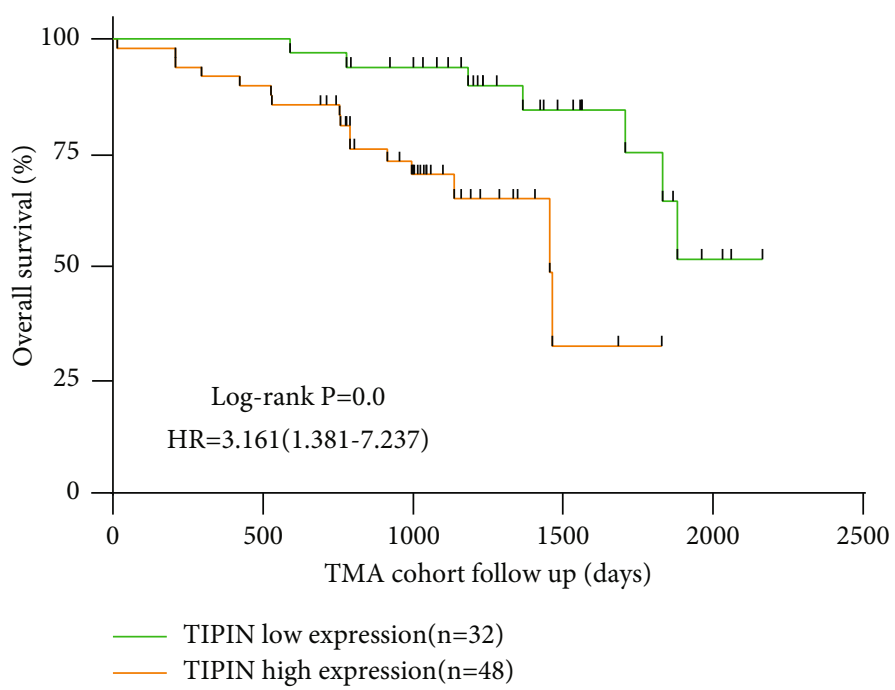

(d)

FIgURE 7: High TIPIN levels were negatively correlated with survival in HCC. Notes: (a) Representative images of TIPIN IHC staining in HCC tissues. (b) Representative TIPIN staining in HCC and adjacent tissues. (c) TIPIN levels are increased in HCC tissues compared to adjacent tissues. (d) Kaplan-Meier analysis shows that upregulated TIPIN levels are correlated with poor prognosis in patients with HCC.

plays an important role in HCC progression [25]. TRIM31 belongs to the tripartite motif-containing or RING, B-box, and coiled-coil family; it acts as a tumor promoter and has been shown to promote HCC progression [26]. However, the relationship between TIPIN or RBM15B and HCC has not been reported. In our study, the data obtained from TCGA and GEO datasets showed the differential expression and prognostic value of the four epigenetically altered driver genes. Simultaneously, it was demonstrated that the RNA expression of TIPIN and RBM15B was discrepant during the development of HCC. Moreover, TMA analysis revealed that TIPIN levels varied between cancerous and paracancerous tissues. Based with clinical information, the prognosis of the patients with high the TIPIN expression was worse than that of those with the low TIPIN expression. TIPIN is associated with TNM stage and could be used as an independent prognostic factor for HCC.

TIPIN interacts with the core circadian protein TIM to form TIM-TIPIN complex, participating in normal DNA replication to maintain genomic stability [27, 28]. During the process of DNA replication, the TIM-TIPIN complex moves along the replication fork through its checkpoint adjustment function or independent adjustment function to maintain the integrity and stability of the replication fork, facilitating the DNA replication process to return to normal $[29,30]$. The downregulation of the TIM-TIPIN complex results in a reduced rate of DNA synthesis [31]. It was previously reported that the TIPIN mRNA level is significantly upregulated in breast cancer, particularly in the most proliferative and poor prognosis-related breast cancer subtypes (triple negative breast cancer, HER2, and Luminal B). Silencing of the TIPIN expression induced apoptosis and inhibited proliferation in breast cancer cell lines, making TIPIN a potential target for breast cancer therapy [32]. Furthermore, knockdown of the TIM-TIPIN complex has been reported to promote apoptosis in melanoma cell lines [33]. However, because of the limited conditions, we could not conduct molecular, cellular, and animal experiments; therefore, the 
TABLE 2: The relationship between TIPIN expression and the clinicopathological features of HCC in TMA.

\begin{tabular}{|c|c|c|c|c|c|}
\hline \multirow{2}{*}{\multicolumn{2}{|c|}{ Clinicopathological features }} & \multirow{3}{*}{$\frac{\text { No. of cases (\%) }}{40}$} & \multicolumn{2}{|c|}{ TIPIN expression level } & \multirow{2}{*}{$P$ value } \\
\hline & & & Low & High & \\
\hline \multirow{2}{*}{ Age (years) } & $\leq 55$ & & 20 & 20 & \multirow{2}{*}{0.068} \\
\hline & $>55$ & 40 & 12 & 28 & \\
\hline \multirow{2}{*}{ Gender } & Female & 17 & 10 & 7 & \multirow{2}{*}{0.074} \\
\hline & Male & 63 & 22 & 41 & \\
\hline \multirow{2}{*}{ Pathogenesis } & $\mathrm{HBV}$ & 58 & 21 & 37 & \multirow{2}{*}{0.261} \\
\hline & Other & 22 & 11 & 11 & \\
\hline \multirow{2}{*}{ ALT (U/L) } & $\leq 35$ & 48 & 20 & 28 & \multirow{2}{*}{0.709} \\
\hline & $>35$ & 32 & 12 & 20 & \\
\hline \multirow{2}{*}{ AST (U/L) } & $\leq 31$ & 42 & 15 & 27 & \multirow{2}{*}{0.411} \\
\hline & $>31$ & 38 & 17 & 21 & \\
\hline \multirow{2}{*}{$\mathrm{TB}(\mathrm{U} / \mathrm{L})$} & $\leq 12$ & 43 & 18 & 25 & \multirow{2}{*}{0.714} \\
\hline & $>12$ & 37 & 14 & 13 & \\
\hline \multirow{2}{*}{$\operatorname{ALB}(\mathrm{G} / \mathrm{L})$} & $\leq 40$ & 47 & 18 & 29 & \multirow{2}{*}{0.711} \\
\hline & $>40$ & 33 & 14 & 19 & \\
\hline \multirow{2}{*}{ TNM } & I and II & 59 & 30 & 29 & \multirow{2}{*}{$0.002^{* *}$} \\
\hline & III and IV & 21 & 2 & 19 & \\
\hline \multirow{2}{*}{ Tumor size } & $\leq 5 \mathrm{~cm}$ & 40 & 18 & 22 & \multirow{2}{*}{0.361} \\
\hline & $>5 \mathrm{~cm}$ & 40 & 14 & 26 & \\
\hline
\end{tabular}

TIPIN: TIM-interacting protein; ALT: alanine amiotransferase; AST: aspartate transaminase; TB: total bilirubin; ALB: albumin; TNM: tumor node metastasis; ${ }^{* *} P<0.01$.

TABLE 3: Univariate and multivariate analysis of the overall survival of HCC in TMA.

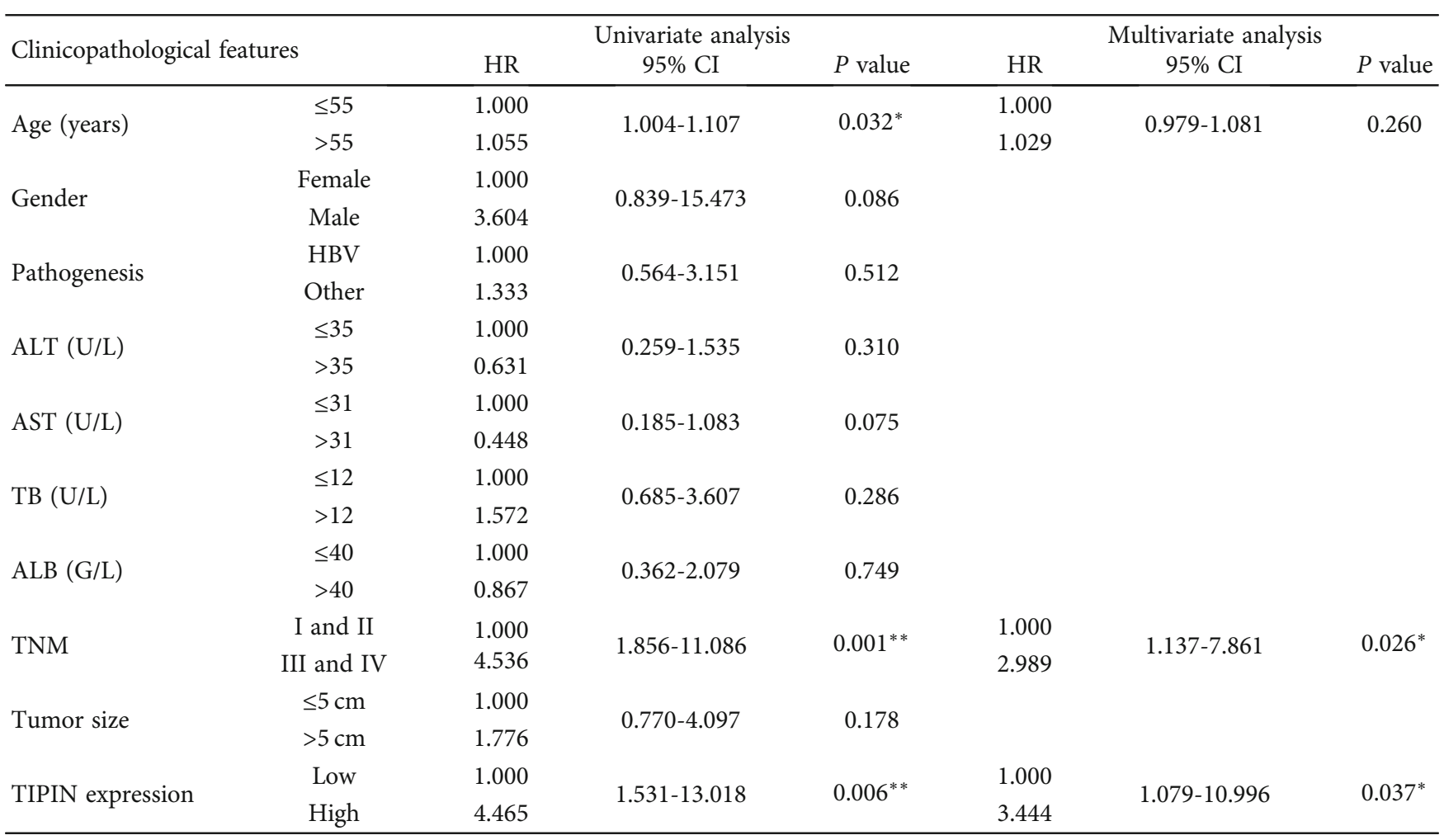

TIPIN: TIM-interacting protein; ALT: alanine amiotransferase; AST: aspartate transaminase; TB: total bilirubin; ALB: albumin; TNM: tumor node metastasis; HR: hazard ratio; CI: confidence interval; ${ }^{*} P<0.05 ;{ }^{* *} P<0.01$. 
specific molecular mechanism of TIPIN in the progression of HCC remains to be further explored.

In conclusion, our study is the first to show that TIPIN is overexpressed in HCC at the mRNA as well as protein level. The high expression of TIPIN indicates a poor prognosis of HCC patients. TIPIN may be a potential prognosis signature for HCC.

\section{Data Availability}

The datasets presented in this study can be found in online repositories. The names of the repository/repositories and accession numbers can be found in the article (TCGA, https:/tcga-data.nci.nih.gov/tcga/;GEO,http://www.ncbi .nlm.nih.gov/geo/; accession numbers: GSE6764, GSE14520, GSE36376, GSE45436, GSE39791, GSE54236, GSE54238, GSE57957, GSE60502, GSE62232, GSE64041, GSE76297, GSE76427, GSE25097, GSE77314, GSE84005, GSE84598, GSE102083, GSE10143, and GSE14811).

\section{Conflicts of Interest}

The authors declare that the research was conducted in the absence of any commercial or financial relationships that could be construed as a potential conflict of interest.

\section{Authors' Contributions}

Zujiang Yu proposed the study. Hui Chen, Chunting Zhang, and Q Zhou performed the research and wrote the first draft. Hui Chen, Chunting Zhang, Yanan Guo, Zhigang Ren, and Qianmei Zhou participated in data analysis and clinical specimen collection. Zujiang Yu is the guarantor. All authors contributed to interpretation of the study and to further drafts. Hui Chen, Chunting Zhang, and Qianmei Zhou contributed equally to this work.

\section{Acknowledgments}

We would like to thank Editage (http://www.editage.cn) for the English language editing.

\section{Supplementary Materials}

Supplementary Table 1 the characteristics of GEO datasets. Supplementary Table 2: univariate and multivariate analysis of the overall survival of HCC in TCGA. Supplementary Figure 1: the negative relationship between promoter methylation level and gene expression level of the expression specific genes. Supplementary Figure 2: ROC curves for evaluating the diagnostic power of TIPIN in HCC. Supplementary Figure 3: molecular mechanism of TIPIN action in HCC. A GSEA of TIPIN based on the Molecular Signatures Database. (Supplementary Materials)

\section{References}

[1] F. Bray, J. Ferlay, I. Soerjomataram, R. L. Siegel, L. A. Torre, and A. Jemal, "Erratum: global cancer statistics 2018: GLOBOCAN estimates of incidence and mortality worldwide for 36 cancers in 185 countries," CA: a Cancer Journal for Clinicians, vol. 70, no. 4, p. 313, 2020.

[2] G. Tian, S. Yang, J. Yuan et al., "Comparative efficacy of treatment strategies for hepatocellular carcinoma: systematic review and network meta-analysis," BMJ Open, vol. 8, no. 10, article e021269, 2018.

[3] J. Yang and J. J. B. Heimbach, "New advances in the diagnosis and management of hepatocellular carcinoma," BMJ, vol. 371, article $\mathrm{m} 3544,2020$.

[4] D. Ban, T. Ogura, K. Akahoshi, and M. Tanabe, "Current topics in the surgical treatments for hepatocellular carcinoma," Annals of Gastroenterological Surgery, vol. 2, no. 2, pp. 137146, 2018.

[5] X. Yang, F. Kong, L. Qi et al., "PARP inhibitor olaparib overcomes sorafenib resistance through reshaping the pluripotent transcriptome in hepatocellular carcinoma," Molecular Cancer, vol. 20, no. 1, p. 20, 2021.

[6] J. M. Llovet, R. K. Kelley, A. Villanueva et al., "Hepatocellular carcinoma," Nature Reviews Disease Primers, vol. 7, no. 1, p. 6, 2021.

[7] P. Farazi and R. A. DePinho, "Hepatocellular carcinoma pathogenesis: from genes to environment," Nature Reviews Cancer, vol. 6, no. 9, pp. 674-687, 2006.

[8] G. Song, X. Zhu, Z. Xuan et al., "Hypermethylation of GNA14 and its tumor-suppressive role in hepatitis B virus-related hepatocellular carcinoma," Theranostics, vol. 11, no. 5, pp. 2318-2333, 2021.

[9] J. Zhang, C. Yang, C. Wu, W. Cui, and L. J. C. Wang, "DNA methyltransferases in cancer: biology, paradox, aberrations, and targeted Therapy," Therapy, vol. 12, no. 8, p. 2123, 2020.

[10] A. Tannapfel, M. Benicke, A. Katalinic et al., "Frequency of p16INK4A alterations and K-ras mutations in intrahepatic cholangiocarcinoma of the liver," Gut, vol. 47, no. 5, pp. 721-727, 2000.

[11] R. Kondratov and M. P. Antoch, "Circadian proteins in the regulation of cell cycle and genotoxic stress responses," Trends in Cell Biology, vol. 17, no. 7, pp. 311-317, 2007.

[12] A. Errico and V. Costanzo, "Differences in the DNA replication of unicellular eukaryotes and metazoans: known unknowns," EMBO Reports, vol. 11, no. 4, pp. 270-278, 2010.

[13] C. Baldeyron, A. Brisson, B. Tesson et al., "TIPIN depletion leads to apoptosis in breast cancer cells," Molecular Oncology, vol. 9, no. 8, pp. 1580-1598, 2015.

[14] M. V. C. Greenberg, "Get out and stay out: new insights into DNA methylation reprogramming in mammals," Frontiers in Cell and Development Biology, vol. 8, p. 629068, 2021.

[15] A. Liu, Q. Wu, D. Peng et al., "A novel strategy for the diagnosis, prognosis, treatment, and chemoresistance of hepatocellular carcinoma: DNA methylation," Medicinal Research Reviews, vol. 40, no. 5, pp. 1973-2018, 2020.

[16] E. Laugsand, F. Skorpen, S. Kaasa et al., "Genetic and nongenetic factors associated with constipation in cancer patients receiving opioids," Clinical and Translational Gastroenterology, vol. 6, no. 6, article e90, 2015.

[17] S. McPherson, M. McMullin, and K. Mills, "Epigenetics in Myeloproliferative Neoplasms," Journal of Cellular and Molecular Medicine, vol. 21, no. 9, pp. 1660-1667, 2017.

[18] M. al-Yozbaki, I. Jabre, N. Syed, and C. M. Wilson, "Targeting DNA methyltransferases in non-small-cell lung cancer," Seminars in Cancer Biology, 2021. 
[19] R. Zhang, Y. Li, H. Yu et al., "An aberrant DNA methylation signature for predicting hepatocellular carcinoma," Annals of Translational Medicine, vol. 8, no. 24, p. 1667, 2020.

[20] J. Low, Q. Tao, K. Ng et al., "A novel isoform of the 8p22 tumor suppressor gene _DLC1_suppresses tumor growth and is frequently silenced in multiple common tumors," Oncogene, vol. 30, no. 16, pp. 1923-1935, 2011.

[21] Y. Zhang, H. Ahsan, Y. Chen et al., "High frequency of promoter hypermethylation ofRASSF1A andp16 and its relationship to aflatoxin B1-DNA adduct levels in human hepatocellular carcinoma," Molecular Carcinogenesis, vol. 35, no. 2, pp. 85-92, 2002.

[22] H. Shiraha, S. Nishina, and K. Yamamoto, "Loss of runtrelated transcription factor 3 causes development and progression of hepatocellular carcinoma," Journal of Cellular Biochemistry, vol. 112, no. 3, pp. 745-749, 2011.

[23] M. J. Baek, Z. Piao, N. Kim et al., "p16 is a major inactivation target in hepatocellular carcinoma," Cancer, vol. 89, no. 1, pp. $60-68,2000$.

[24] E. Abeni, A. Salvi, E. Marchina, M. Traversa, B. Arici, and G. de Petro, "Sorafenib induces variations of the DNA methylome in HA22T/VGH human hepatocellular carcinomaderived cells," International Journal of Oncology, vol. 51, no. 1, pp. 128-144, 2017.

[25] O. Okochi, K. Hibi, M. Sakai et al., "Methylation-mediated silencing of SOCS-1 gene in hepatocellular carcinoma derived from cirrhosis," Clinical Cancer Research, vol. 9, no. 14, pp. 5295-5298, 2003.

[26] H. Yoshikawa, K. Matsubara, G. Qian et al., "SOCS-1, a negative regulator of the JAK/STAT pathway, is silenced by methylation in human hepatocellular carcinoma and shows growthsuppression activity," Nature Genetics, vol. 28, no. 1, pp. 29$35,2001$.

[27] X. Liu, X. Hu, Y. Kuang et al., "BCLB, methylated in hepatocellular carcinoma, is a starvation stress sensor that induces apoptosis and autophagy through the AMPK-mTOR signaling cascade," Cancer Letters, vol. 395, pp. 63-71, 2017.

[28] D. Wang, S. Han, R. Peng et al., "DUSP28 contributes to human hepatocellular carcinoma via regulation of the p38 MAPK signaling," International Journal of Oncology, vol. 45, no. 6, pp. 2596-2604, 2014.

[29] P. Guo, X. Ma, W. Zhao et al., "TRIM31 is upregulated in hepatocellular carcinoma and promotes disease progression by inducing ubiquitination of TSC1-TSC2 complex," Oncogene, vol. 37, no. 4, pp. 478-488, 2018.

[30] A. Errico and V. Costanzo, "Mechanisms of replication fork protection: a safeguard for genome stability," Critical Reviews in Biochemistry and Molecular Biology, vol. 47, no. 3, pp. 222-235, 2012.

[31] A. Leman and E. Noguchi, "Local and global functions of Timeless and Tipin in replication fork protection," Cell Cycle, vol. 11, no. 21, pp. 3945-3955, 2012.

[32] T. Chen, P. Stephens, F. Middleton, and N. J. Curtin, "Targeting the S and G2 checkpoint to treat cancer," Drug Discovery Today, vol. 17, no. 5-6, pp. 194-202, 2012.

[33] A. Chakraborty, F. Aziz, E. Roh et al., "Knock-down of the TIM/TIPIN complex promotes apoptosis in melanoma cells," Oncotarget, vol. 11, no. 20, pp. 1846-1861, 2020. 